www.jmscr.igmpublication.org

Impact Factor 5.84

Index Copernicus Value: 71.58

ISSN (e)-2347-176x ISSN (p) 2455-0450

crossref DOI: _https://dx.doi.org/10.18535/jmscr/v5i11.30

Journal Of Medical Science And Clinical Research

\title{
Assesment of Outcome of Using Syndesmotic Screws in Ankle Injuries
}

\section{Authors \\ Nithyakumar.V.R ${ }^{1}$, Neelakrishnan. $R^{2}$., Barathiselvan. ${ }^{3}$, Shanmugaraja. A. $T^{4}$ Harishkumar. $\mathbf{R}^{5}$}

${ }^{* 1}$ Postgraduate in Orthopaedics, Rajah Muthiah Medical College \& Hospital, Annamalai University ${ }^{2}$ Professor \& HOD of Orthopaedics, Rajah Muthiah Medical College \& Hospital, Annamalai University ${ }^{3}$ Senior Resident in Orthopaedics, Rajah Muthiah Medical College \& Hospital, Annamalai University

4,5 Lecturer, Department of Orthopaedics, Rajah Muthiah Medical College \& Hospital, Annamalai University

\begin{abstract}
Introduction: In a predicted 1-11\% of all ankle sprains, injury of the distal tibiofibular syndesmosis happens. $40 \%$ of patients have ankle instability 6 months after an ankle sprain. This might be because of widening of the ankle mortise as a result of stretch of syndesmotic ligaments after acute ankle sprain. In fractures of the ankle, syndesmotic damage takes place in approximately 50\% of Weber type B and in all of
\end{abstract} Weber type $C$ fractures.

Aim: A Study to analyse the importance of the using syndesmotic screws in ankle injuries.

Materials \& Methods: All cases of ankle injuries were assessed for syndesmotic disruption and finally taken up for surgery and Follow up achieved at regular interval for durations of 1 year. Post operatively patients were assessed using olerund and mollander scoring system.

Results: Twenty patients with syndesmotic disruption in ankle injuries had been treated with stainless steel syndesmotic screws and subsequently removed in 8-12 weeks then allowed for weight bearing have been studied from june 2015 to September 2017. Mean age of the patients 45.75 years (23-65 years). Mean hospital stay was 14 days (ranging from 12 to 17 days). The mean Olerud and Mollander score is 80.75(7095).

Conclusion: In our study, Patients with syndesmotic screws had results that were comparable with that of other research done elsewhere with good functional outcome.

Keywords: syndesmotic disruption, syndesmotic screws.

\section{Introduction}

Syndesmosis is described as a fibrous joint wherein adjacent bones are related by means of a strong ligaments. This definition also applies for the distal tibiofibular syndesmosis, which is a syndesmotic joint composed of two bones and 4 ligaments. The distal tibia and fibula share the osseous part of the syndesmosis and are linked via the distal anterior tibiofibular ligament (ATIFL), the distal posterior tibiofibular ligament (PTIFL), the transverse ligament and the interosseous ligament. Although the syndesmosis is a joint, within the literature the term syndesmotic injury is used to explain injury of the syndesmotic ligaments.

In an estimated $1-11 \%$ of all ankle sprains, injury of the distal tibiofibular syndesmosis takes place. $40 \%$ of patients still complaining of ankle instability 6 months after an ankle sprain. This might be because of widening of the ankle mortise 
as a result of expanded size of the syndesmotic ligaments after acute ankle sprain. As widening of the ankle mortise through $1 \mathrm{~mm}$ increases the space of the tibiotalar joint by $42 \%^{(1)}$ this will cause instability and therefore early osteoarthritis of the tibiotalar joint.

Syndesmotic disruption can occur in ankle injuries, with or without a fracture of the osseous element. In fractures of the ankle, syndesmotic get injured in about $50 \%$ of Weber type B and in all Weber type $\mathrm{C}$ fractures, whereas in ankle sprains without fracture, syndesmotic disruption accounts for $1-11 \%$ of all ankle trauma ${ }^{(2)}$.

However, in discussing syndesmotic injury it seems the exact proximal and distal borders of the distal tibiofibular syndesmosis are not properly described.

There is no clear assertion in etiological ${ }^{(3)}$, genetic $^{(4)}$ or topographical ${ }^{(5)}$ fracture classifications, regarding the exact volume of the syndesmosis. Syndesmotic joint is now not in reality described in anatomical textual content books ${ }^{(6)}$ Kelikian $^{(7)}$ postulate that the distal - joint starts origin at the level of the tibiofibular ligaments from the tibia and ends where those ligaments insert into the fibular malleolus.

\section{Materials and Methadology}

This is a prospective, time bound hospital based study performed in Rajah Muthiah Medical College, Chidambaram between July 2015 to September 2017. This study consist of 20 patients of syndesmotic disruption along with variable ankle fracture, who were evaluated preoperatively and intervened with appropriate fracture fixation and fixed with syndesmotic screws. Depends on the need of stability, number of screws and variety of cortical purchases had been determined intra-operatively. All patients were informed about the study and informed written consent become received.

\section{Inclusion Criteria}

a) Patient who have been recognized to have distal tibiofibular syndesmotic disruption based on clinical and radiological strategies. b) Age group among 18 and sixty five years.

c) Patient presented with less than 1 month old trauma.

d) Patient who have medical co -morbidities under control.

e) Patients who were medically fit for surgical procedure.

f) Patient who had been willing for the study and surgery.

\section{Exclusion Criteria}

a) Patients less than 18 years and more than 65 years.

b) Patients who had greater than 1 month old trauma

c) Patients who have uncontrollable medical comorbidiies.

d) Patients who were medically not fit for surgery

e) Patients who had been not willing to undergo surgery or study

\section{Data Collection and Analysis}

After the patient who have been identified with distal tibiofibular syndesmotic disruption was admitted after preliminary stabilisation with below knee slab. All the necessary medical information had been recorded within the profoma prepared for this study. All the cases with diagnosed syndesmotic disruption with fractures had been treated by means of syndesmotic screw $(4 \mathrm{~mm}$ cancellous screw) fixation. In doubt full cases, to begin with the fractures have been fixed after which by means of performing cotton hook test, after confirming syndemotic disruption, it was fixed with $4 \mathrm{~mm}$ cancellous screws. Number of cortices to be purchased and number of screws needed was decided intra-operatively depending at the need of stability of disruption. Intra-operative datas recorded in the profoma. After completing the hospital treatment, patients were discharged and called for regular follow-up at 1,3,6,12 and 18 months. Meanwhile the need of screw removal will be decided at 3 months and dealt with therefore relying on the pain and restricted dorsiflexion and subsequently allowed for weight bearing. 
All the patients have been clinically assessed by Olerud and Molander scoring system at 3 months interval. Radiological assessment for progression and time of union, fracture alignment and implant related complications have been analysed. Data collected at the end of the study was statistically analysed.

\section{Diagnostic Criteria for Syndesmotic Disruption}

As in all ankle injuries, physical examination should involve a systematic approach. By palpation of the malleoli and other bony landmarks, consisting of the proximal fibula, fundamental ligamentous regions need to be assessed to exclude related issues. Many assessments were described. Apart from diagnostic check there are some standards which confirms syndesmotic disruption radiologically. They are

1) Fracture of posterior malleoli

2) Increase in medial clear apace

3) Disruption of ankle mortise

4) Anterior inferior tibio fibular ligament disruption proven with the aid of decrease in tibio fibular overlap

Other test are as follows: In the squeeze check, pain is elicited over the ankle joint as the distal tibia and fibula separate while the mid calf of the leg or just above is compressed. In the external rotation test, the mechanism of disruption is reproduced with the ankle dorsiflexed and foot externally rotated: This test can be positive if the affected person complains of pain. The test may also be undertaken with the affected person standing and rotating the body with the foot at the ground. Pain over the anterolateral region of the ankle joint elicited in passive dorsiflexion may also help one to suspect a syndesmotic damage but isn't specific.

The fibular translation test, the Cotton test, and the crossover leg test have been additionally described $^{(8)}$. In the fibular translation test, the examiner tries to move the fibula in the anteriorposterior plane: An increased translation, in comparison with the opposite side indicative of a syndesmosis injury.
In the Cotton hook test, the talus is translated in the mortise within the medial-lateral plane: Increased translation and pain suggest a positive test. In the crossed-leg test, the affected person is seated with the mid-fibula of the injured leg on the knee of the uninvolved leg; the involvement of the syndesmotic injury is suspected if pain is felt when the knee of the affected leg is driven closer to the ground $^{(9)}$. Standard radiographs of the ankle, comprising weight-bearing anteroposterior, mortise, and lateral views, have to be the first set of investigations while a syndesmosis damage is suspected. A tibiofibular clear space greater than 6 $\mathrm{mm}$ located $10 \mathrm{~mm}$ above the plafond is suggestive of syndesmosis damage, while increase in medial clear space between the medial malleolus and the talus can be suggestive of syndesmotic and deltoid ligament disruption ${ }^{(10)}$.

Finally all injured ankles were examined the use of intraoperative fluoroscopic external rotation stress assessments. The contralateral limb is used as a control. A external rotation force is carried out for stress examination. Stress test was repeated after lateral malleolar fixation and repeated after medial malleolar and syndesmotic fixation ${ }^{(11)}$.

\section{Management of the Patient}

As soon as the patient become admitted, a detailed history was taken and a meticulous examination of the patients was achieved. The informations were recorded in the profoma organized. X-ray ankle anteroposterior view, Mortise view and lateral view have been taken. Depending at the increase in medial clear space and reduced tibiofibular overlap, similarly the syndesmotic disruption become confirmed by means of stress dorsiflexion view. Further the fracture was classified according to Lauge Hansen classification. Only the fracture with syndesmotic disruption included in the study. Below knee slab applied and the limb was kept in Bohler braun splint until the patient became taken up for surgery. 


\section{Surgical Technique}

Surgical management was done by experts. Since initial priority given to ankle fracture, implants needed for them must be considered. For fixing the fibula the implants needed are 1/3rd tubular plate, asian DCP, recon plate with appropriate screws. For fixing the medial malleolus k-wire with tension band wiring, malleolar screws. In case of trimalleolar fracture posterior malleoli also ought to be taken into consideration. Regarding the syndesmotic fixation stainless steel $4 \mathrm{~mm}$ cancellous screws of variable length is needed. Patient positioned supine in fracture table with wedge beneath ipsilateral buttock, with tourniquet control and c-arm guidance.

Incision made over the lateral aspect of leg centred over fibula fracture. Hematoma was cleared on the fracture site, then fracture reduced and fixed with one third tubular plate. The one third tubular plate is the work horse plate of distal fibula that is low profile and provide sufficient power for most fractures. In our observe most of the case (18) patients, the fibula fixation was done with the aid of one-third tubular plate, in one patient reconstruction plate become used. In one patient there is no fibula fracture.

Medial malleoli exposed after putting off the wedge under the buttock. Hematoma was cleared from fracture site. Any soft tissue interposition found will be cleared from fracture site. Fracture reduced with the use of point reduction clamp and then stabilised with malleolar screw or k-wire and tension band wiring. In our study out of 20 patients 16 patients had been fixed with malleolar screw and a couple of patients had been fixed with k-wire and tension band wiring. In 2 patients deltoid ligament got injured, for this deltoid ligament reconstruction was done using 1-0 prolene.

In one case posterior malleoli additionally got fractured, for this reason patient postion turned into floppy lateral function and fixed with malleolar screw with washer.

\section{Fixation of Syndesmotic Disruption}

Repositioning of the patient with wedge below ipsilateral buttock and flouroscopy is required inorder to ensure that the screws are inserted at the proper level and are positioned parallel to the joint line; - if a lateral incision is require to fix a concomitant lateral malleolar fracture, then the incision was made more posteriorly inorder to facilitate syndesmotic screw insertion. Patients needed to be warned that hardware failure (screw breakage) is a common complication and does not imply surgical error.

\section{Proper Level of Syndesmotic Screw}

Place the first screw about $1 \mathrm{~cm}$ proximal to syndesmosis or $4 \mathrm{~cm}$ proximal to the ankle joint. If it's far too low, it can skip via the distal tibiofibular articulation (or may skip via interosseous ligament) that may localized calcification and/or pain. If the screw insertion is simply too high, it will make the tip of the fibula to toe outward. It is necessary to direct the screws parallel to the joint line inorder to avoid tilting the distal fibula, following insertion, get fluoroscopy images to rule out malreduction of the fibula in notch of tibia, with inferior and anterior subluxation of talus. In some cases the syndesmotic screws were located too low which may also lead to persistent ankle pain (specially if the screw breaks inside the tibial-fibular joint). Screw is inserted obliquely from posterior to anterior at angle $25 \mathrm{deg}$ to 30 degree. It is directed from lateral to the medial cortex of the fibula, and into lateral cortex of the tibia at right angles to the long axis.

It is crucial that the screw also be directed parallel to the joint line. If the screw is directed proximally or distally there may be shortening or lengthening on the fracture site.

\section{Results}

During the period of study a total of 20 patients were received with distal tibio fibular syndesmotic disruption with variable type of ankle fractures. All these patients were evaluated clinically and radiologically and after diagnosed with syndesmotic disruption, initially managed with 


\section{JMSCR Vol||05||Issue||11||Page 29988-29996||November}

below knee splint and limb elevation then subsequently planned for surgery. All patients were operated by fixing of syndesmotic disruption with stainless steel screws.

Average age of the patient was 45.75 years (range 23-65 years). There are 12 male patients and 8 female patients. The youngest patient in our study was 23 years old and the oldest was 60 years old. 12 fractures involved right side and 8 patients has left sided injury. Fracture types were given in the table 1. The most common mode of injury was road traffic accident involved 13 patients and 7 patients by self fall. Average trauma surgery interval was 6 days (range 2-10 days). Average surgical time was 59 minutes (range 50-75 mins). Average blood loss was $104 \mathrm{ml}$ (range 80-150 $\mathrm{ml}$ ). Average hospital stay was 14 days (range 12-
16 days). Single syndesmotic screw was used in 18 patients and two screws are used in 2 patients. Tricortical purchase was tried in 18 patients and quadricortical purchase was tried in 2 patients. Screws removed around 3 months in 6 patients, whole implant was removed in 6 patients till then allowed was weight bearing with screws. Till 8 patients were on regular followup, among them 4 patients was operated 6 months back, 4 patients were waiting for full implant removal without complication. In our study there were no screw breakage complication. Minor complications like infection, persistent pain and screw backout were encountered. Which were managed appropriately. In long term follow up these patients shows no significant morbidity. Average olerud and molander scoring was 80.75 (range 70-95).

\section{Case 1}
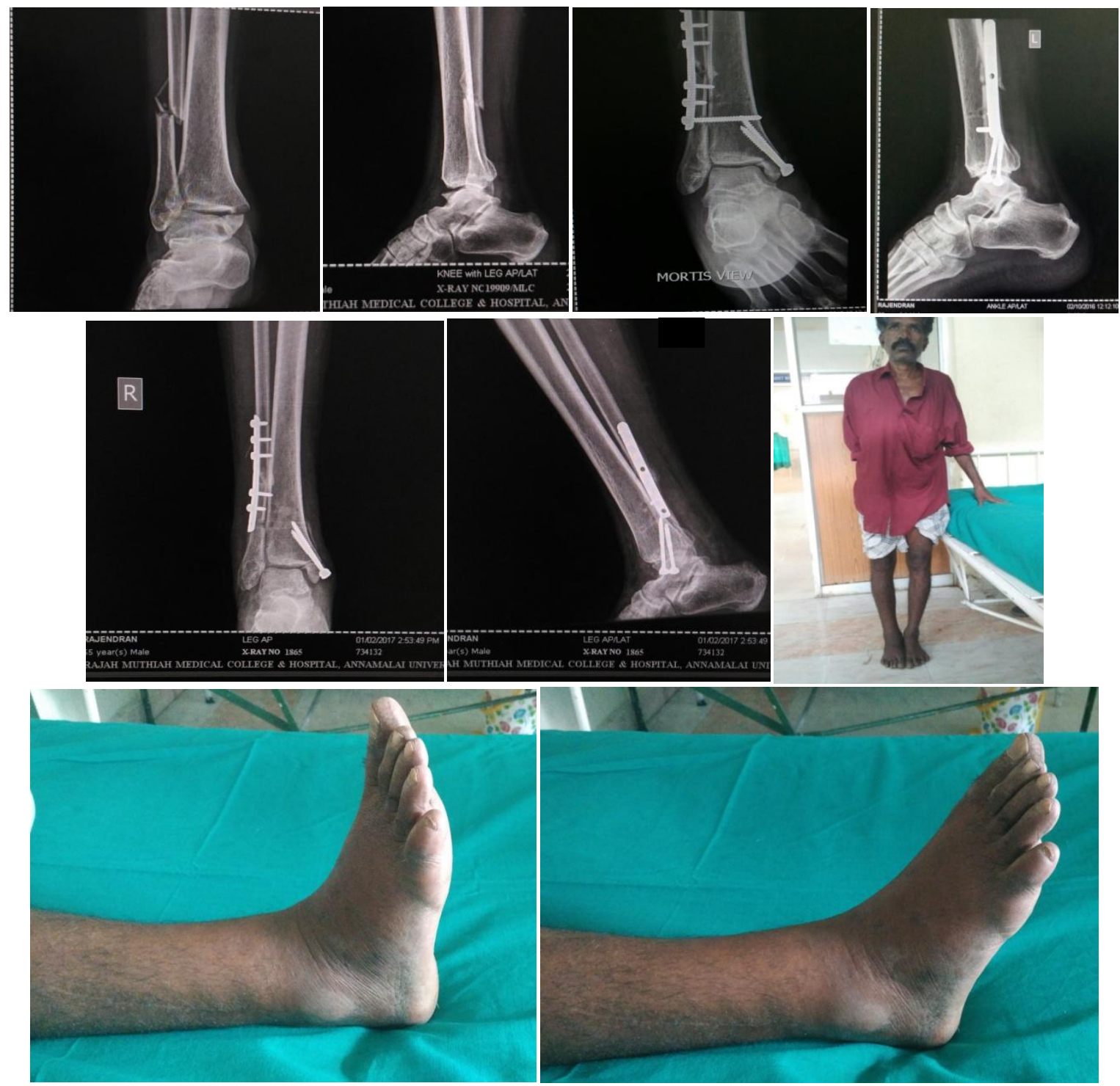


\section{JMSCR Vol||05||Issue||11||Page 29988-29996||November}

\section{Case 2}

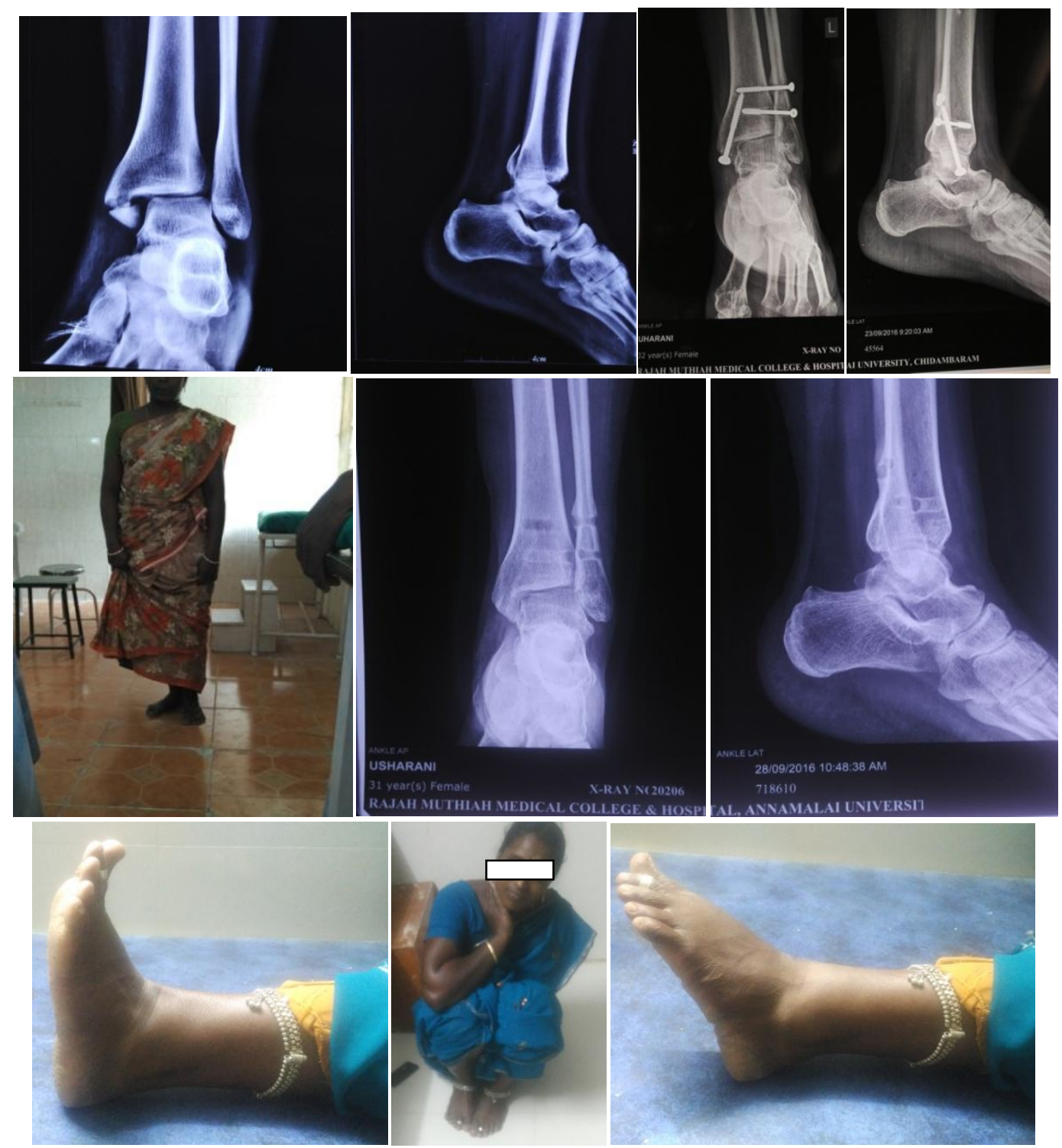

Table 1: type of fracture

\begin{tabular}{|l|c|}
\hline Type of fracture & Number of patient \\
\hline PER II & 1 \\
\hline PER III & 14 \\
\hline PER IV & 3 \\
\hline SER IV & 2 \\
\hline
\end{tabular}

Table 2: Olerud and Molander score

\begin{tabular}{|l|c|}
\hline Score & Number of patient \\
\hline Excellent & 4 \\
\hline Good & 11 \\
\hline Fair & 5 \\
\hline
\end{tabular}

Table 3: Complications

\begin{tabular}{|l|l|}
\hline Post operative infection & 1 \\
\hline Persistent ankle pain & 1 \\
\hline Screw backout & 1 \\
\hline Valgus angulation & 1 \\
\hline Late diastasis & 0 \\
\hline Tibio fibular synostosis & 0 \\
\hline
\end{tabular}

\section{Discussion}

Our study consists of 20 cases of closed ankle fractures with syndesmotic disruption diagnosed by radiological views and intra-operative tests. Maximum incidence of the injury was in the fifth decade of life. Injury was more common in males$12(60 \%)$ and females being 8(40\%). Right side was more commonly involved-12 patients (60\%).

Road traffic accidents contributed to $65 \%$ of injuries, followed by self fall while walking (35\%). Out of 20 patients, 14 are PER TYPE III pattern, 3 patients are PER TYPE IV pattern, 1 cases of PER TYPE II pattern and 2 cases of SER TYPE IV.

The most common injury pattern seen in our study was Pronationexternal rotation type III. Stress 
radiographs are useful to assess ankle instability. schonk et al suggested that gravity stress test is comfortable and more sensitive than manual stress test. Weber stated that instability is overestimated by stress radiographs. Evaluation of deep deltoid ligament injury associated with ankle instability is assessed by stress radiographs.

Pronation external rotation injury type III and IV was very unstable and also associated syndesmotic disruption which should be diagnosed clinically and radiologically and must be fixed with syndesmotic screw from fibula to tibia, which may be stabilised by tricortical or quadricortical fixation depends on pattern of injury.

Functional outcome was equal for both tricortical and quadricortical fixation supported by Macleod mark et $\mathrm{al}^{(12)}$. But in fear of chance of screw breakage in quadricortical screw we usually prefer tricortical fixation. In case of need of more stability we usually prefer two tricortical screw instead of single quadricortical screw to avoid screw breakage.

Among 20 patients, at the end of six months 4 patients (20\%) shows excellent outcome, 11 patients $(55 \%)$ shows good outcome and 5 patients shows fair outcome with minimal complications which subsides later. After six months followup in some cases, the patients who showed good outcome improved to excellent outcome with rehabilitation.

Among 20 patients, one patient had early screw backout most probably due to osteoporosis, then we treated by screw removal. This patient had no complication further. One patient had persistent ankle pain after screw removal, most probably due to early screw removal at 8 weeks post-operative period. She was followed periodically at 3 months interval to find radiological evidence of ankle arthritis. Till now this patient had no radiological feature of ankle arthritis, also the pain was reduced subsequently. Another patient had superficial infection for about 4 weeks postoperatively which was treated by appropriate antibiotics and high protein diet, then the wound healed completely.
To avoid these complications, in case of osteoporotic bones, we prefer two tricortical screws to prevent screw backout. To avoid ankle pain we prefer delayed screw removal depends on restriction of dorsiflexion, and also started weight bearing with screws insitu. Till now we not yet faced screw breakage. Still we have 3 patients of more than one and half year post-operative period who refused implant removal surgery. They were followed periodically every 6 months by home visit to check for any complaints while weight bearing. All the three patients doing well upto date. We were planning for implant removal for these three patient to avoid unnecessary complications. David paul bell et $\mathrm{al}^{(13)}$ study shows maximum of three years of patient with retained screw without complications

In pronation external rotation injury fibular length restoration and rotation, ankle mortise and syndesmotic stability is important factor as noted by maverick et $\mathrm{al}^{(14)}$. We had good to excellent results in all cases as we could maintain the syndesmotic stability and fibular length by syndesmotic screws and fibular plating. Displacement is position of talus in the mortise and depends on intact deep deltoid ligament ${ }^{(15)}$.

Fixing only the malleolar fragment will not restore ankle stability in case of deltoid ligament rupture. It must be repaired subsequently if the deep deltoid ligament is torn ${ }^{(16)}$. Stable fractures shows no displacement in axial loading ${ }^{(17)}$. Treatment decisions are based on the stability of fracture. Prognosis is determined by energy of injury. Fixing the malleolar fragment will not restore ankle stability and need to repair deep deltoid if torn. Even though Lauge-Hansen classification describes in detail about the pattern of ankle fracture it does not deal with syndesmotic injuries According to Micheal Bekorom ${ }^{(18)}$, pronation injuries/weber type $\mathrm{C}$ fractures are commonly associated with syndesmotic injuries than supination injuries/weber type B injuries, our study also reflects similar incidence of syndesmotic injury among the various fracture pattern. 
Miller study shows the functional outcome of ankle fractures with syndesmotic screw fixation was ranging from 75-85 of ollerud and mollander scoring system. ${ }^{(19)}$ Our study shows $20 \%$ of excellent result with score of 95 and $55 \%$ shows good result with score of $81-90,25 \%$ shows fair results with some complications which were treated subsequently..

\section{Conclusion}

Though pronation and supination injuries produces syndesmotic disruption, we encounter most commonly the pronation-external rotation injury. In that PER TYPE III and IV are commonly seen. Though there are many controversies regarding need to fix syndesmotic disruption or not, our study concludes there is definitive need of fixation of syndesmotic disruption that too by syndesmotic screw. Though there are various methods of fixation, in our study fixing with screws shows good functional outcome. Regarding the amount of cortical purchase we prefer tricortical purchase, which will avoid the implant breakage and helps in delayed implant removal as a whole, which helps to avoid multiple surgeries for implant removal.

Some studies shows there is chance of screw breakage, we not yet faced breakage as we prefer tricortical fixation. In view of stability tricortical fixation gives excellent stability, in few cases quadricortical fixation was attempted and in that cases implant was removed at appropriate time. In few case two screws were attempted to increase the stability, and implant was removed as whole in later date shows excellent functional outcome.

We faced some complications like persistent ankle pain due to early screw removal, infection, and screw backout due to osteoporotic patient, on subsequent follow up and appropriate treatment these complications subsides on later date. Though age and sex of the patient, type of fracture, time of presentation, amount of blood loss, time to union influences the functional outcome, these parameters shows no gross variations in all patients regarding outcome and showed good result.

As a whole for syndesmotic disruption in ankle injuries, there must be a need of fixation of distal tibio fibular syndesmosis, that too by syndemotic screws shows excellent functional outcome, and by tricortical fixation reduces untoward complications.

\section{References}

1. Ramsey PL, Hamilton W. Changes in tibiotalar area of contact caused by lateral talar shift. J Bone Joint Surg Am. 1976;58:356-357

2. Hopkinson WJ, St Pierre P, Ryan JB, et al. Syndesmosis sprains of the ankle. Foot Ankle. 1990;10:325-330.

3. Ashhurst APC, Bromer RS. Classification and mechanism of fractures of the leg bones involving the ankle: based on a study of three hundred cases from Episcopal Hospital. Arch Surg. 1922;4:51129.

4. Lauge-Hansen N. Fractures of the ankle. II. Combined experimental-surgical and experimental-roentgenologic investigations. Arch Surg. 1950;60:957-985

5. Weber BG. Die Verletzungen des oberen Sprunggelenkes. Bern: Hans Huber; 1972. pp. 51-65

6. Lanz VT, Wachsmuth W. Praktische Anatomie. Berlin: Springer-Verlag; 1972. pp. 328-360

7. Kelikian H, Kelikian S. Disorders of the Ankle. Philadelphia: W.B. Saunders Company; 1985. pp. 4-8

8. Jenkinson, Richard J MD; Sanders, David W MD, MSc, FRCS(C); Macleod, Mark D MD, FRCS(C); Domonkos, Andrea BSc; Lydestadt, Jeanette RNJournal of Orthopaedic Trauma: October 2005 Volume 19 - Issue 9 - pp 604-609doi: 10.1097/01.bot.0000177114.13263.12

9. McCollum $\mathrm{G}$, van den Bekerom $\mathrm{M}$, Kerkhoffs G, Calder J, van Dijk C. 
Syndesmosis and deltoid ligament injuries in the athlete. Knee Surg Sports Traumatol Arthrosc. 2013;21:1328-1337. doi: 10.1007/s00167-012-2205-1.

10. Beumer A, Swiestra B, Moulders P. Clinical diagnosis of syndesmosis instability: evaluation of stress tests behind the curtains. Acta Orthop Scand. 2002; 73:667-669.

11. Cesar de Cesar P, Muller E. Comparison of MRI to physical examination for syndesmotic injuries after lateral ankle sprain. Foot Ankle Int. 2011;32:10-16. doi: 10. 3113/FAI. 2011.1110.

12. Manjoo, Ajay MD; Sanders, David W MD, MSc, FRCSC; Tieszer, Christina MSc; MacLeod, Mark D MD, FRCSC Journal of Orthopaedic TraumA doi: 10.1097/BOT.0b013e3181a9f7a5

13. David Paul Bell, Merng Koon Wong ${ }^{\mathrm{b}}$ Department of Orthopaedic Surgery, Changi General Hospital, 2 Simei Street 3, Singapore 529889, Republic of Singapore, Accepted 1 February 2006, Available online 19 April 2006

14. Yablon I.G., Segal .D., Leanch R.E., Ankle Injuries, New york, 1983, Churchill Livingstone.

15. Yablon I.G., Heller. F.G., Shouse .L; The Key role of lateral malleolus in displaced fractures of ankle: J. Bone Joint Surg : 59 A: 169, 1977.

16. S.Terry canale,James H Beauty Campbell's operative orthopaedics, ed12, 2013, Mosby Company

17. Journal of college of Medical sciencesNepal, Early weight bearing compared with non-weight bearing functional mobilization after operative treatment of an ankle fracture, 2011, vol-7,No.-1, 4046 ,

18. Michel P.J Vanden bekerom, WJO, 2011, July 18, 2(7);51-56.

19. Miller, Anna N MD*; Paul, Omesh MD*; Boraiah, Sreevathsa MD†; Parker, Robert
J BS*; Helfet, David L MD*; Lorich, Dean G MD* Journal of Orthopaedic Trauma: January 2010 - Volume 24 - Issue 1 - pp 12-16. 